\title{
A REPRESENTATION FORMULA RELATED TO SCHRÖDINGER OPERATORS
}

\author{
SHIJUN ZHENG
}

\begin{abstract}
Let $H=-d^{2} / d x^{2}+V$ be a Schrödinger operator on the real line, where $V \in L^{1} \cap L^{2}$. We define the perturbed Fourier transform $\mathcal{F}$ for $H$ and show that $\mathcal{F}$ is an isometry from the absolute continuous subspace onto $L^{2}(\mathbb{R})$. This property allows us to construct a kernel formula for the spectral operator $\varphi(H)$.
\end{abstract}

Schrödinger operator is a central subject in the mathematical study of quantum mechanics. Consider the Schrödinger operator $H=-\triangle+$ $V$ on $\mathbb{R}$, where $\triangle=d^{2} / d x^{2}$ and the potential function $V$ is real valued. In Fourier analysis, it is well-known that a square integrable function admits an expansion with exponentials as eigenfunctions of $-\triangle$. A natural conjecture is that an $L^{2}$ function admits a similar expansion in terms of "eigenfunctions" of $H$, a perturbation of the Laplacian (see 7. Ch.XI and the notes), under certain condition on $V$.

The three dimension analogue was proven true by T.Ikebe [6], a member of Kato's school, in 1960. Later his result was extended by Thor to the higher dimension case [10]. In one dimension, recent related results can be found in e.g., Guerin-Holschneider [5], Christ-Kiselev [4] and Benedetto-Zheng [3].

Throughout this paper we assume $V: \mathbb{R} \rightarrow \mathbb{R}$ is in $L^{1} \cap L^{2}$. We shall prove a one-dimensional version of Ikebe's theorem for $L^{2}$ functions (Theorem 1). Theorem 2 presents an integral formula for the kernel of the spectral operator $\varphi(H)$ for a continuous function $\varphi$ with compact support. In a sequel to this paper we shall use this explicit formula to study function spaces associated with $H$ (see [3]).

The generalized eigenfunctions $e(x, \xi), \xi \in \mathbb{R}$ of $H$ satisfy

$$
\left(-d^{2} / d x^{2}+V(x)\right) e(x, \xi)=\xi^{2} e(x, \xi)
$$

in the sense of distributions.

Date: November 9, 2018.

2000 Mathematics Subject Classification. Primary: 42C15; Secondary: 35P25.

Key words and phrases. spectral theory, Schrödinger operator.

The author is supported in part by DARPA (Defense Advanced Research Projects Agency). 
Definition. The perturbed Fourier transform $\mathcal{F}$ on $L^{2}$ is given by

$$
\begin{aligned}
\mathcal{F} f(\xi) & =\text { l.i.m. }(2 \pi)^{-1 / 2} \int f(x) \overline{e(x, \xi)} d x \\
= & \lim _{N \rightarrow \infty}(2 \pi)^{-1 / 2} \int_{-N}^{N} f(x) \overline{e(x, \xi)} d x,
\end{aligned}
$$

where the convergence is in $L^{2}$ norm as $N \rightarrow \infty$. By Theorem $1, \mathcal{F}$ is a well-defined isometry from $\mathcal{H}_{a c}$ onto $L^{2}$.

Theorem 1. Suppose $V \in L^{1} \cap L^{2}$. Then there exists a family of solutions $e(x, \xi),|\xi| \in[0, \infty) \backslash \mathcal{E}_{0}, \mathcal{E}_{0}$ being a bounded closed set of measure zero, to equation (11) with the following properties.

(i) If $f \in L^{2}$, then there exists an element $\tilde{f} \in L^{2}$ such that

$$
\mathcal{F} f(\xi)=\tilde{f}(\xi) \quad \text { in } L^{2} .
$$

(ii) The adjoint operator $\mathcal{F}^{*}$ is given by

$$
\mathcal{F}^{*} g=\text { l.i.m. } \text {. }_{\rightarrow \infty} \sum_{i=1}^{N}(2 \pi)^{-1 / 2} \int_{\alpha_{i} \leq \xi^{2} \leq \beta_{i}} g(\xi) e(x, \xi) d \xi
$$

in $L^{2}$, where $\left[\alpha_{i}, \beta_{i}\right) \subset(0, \infty)$ are a countable collection of disjoint intervals with $[0, \infty) \backslash \mathcal{E}_{0}^{2}$ equal to $\cup_{i}\left[\alpha_{i}, \beta_{i}\right)$.

(iii) If $f \in L^{2}$, then $\left\|P_{a c} f\right\|_{L^{2}}=\|\tilde{f}\|_{L^{2}}$, where $P_{a c}$ is the projection onto $\mathcal{H}_{a c}$, the absolute continuous subspace in $L^{2}$.

(iv) $\mathcal{F}: L^{2} \rightarrow L^{2}$ is a surjection. Moreover, $\mathcal{F F}^{*}=I d$ and $\mathcal{F}^{*} \mathcal{F}=P_{a c}$.

(v) If $f \in \mathcal{D}(H)$, then $(H f)^{\sim}(\xi)=\xi^{2} \tilde{f}(\xi)$ in $L^{2}$.

Remark 1. The proof is based on the ideas of [6] for 3D. We also use some simplifications as found in Reed and $\operatorname{Simon}\left(\mathbf{1}^{7}\right)$ and Simon [8].

Remark 2. If $|e(x, \xi)| \leq C$ a.e. $(x, \xi) \in \mathbb{R}^{2}$, then we have a "betterlooking" form in $(i i)$ of the theorem

$$
\mathcal{F}^{*} g=\text { l.i.m. }(2 \pi)^{-1 / 2} \int g(\xi) e(x, \xi) d \xi
$$

If $H=\int \lambda d E_{\lambda}$ is the spectral resolution of $H$, define the spectral operator $\varphi(H):=\int \varphi(\lambda) d E_{\lambda}$ by functional calculus. We prove a representation formula for the integral kernel of $\varphi(H)$. 
Let $\left\{e_{k}\right\}_{k=1}^{\infty}$ be an orthonormal basis in $\mathcal{H}_{p}$, the subspace of eigenfunctions in $L^{2}$ for $H$ and let $\lambda_{k}$ be the eigenvalue corresponding to $e_{k}$.

Theorem 2. Let the operator $H$ be as in Theorem 1. Suppose $\varphi: \mathbb{R} \rightarrow$ $\mathbb{C}$ is continuous and has a compact support disjoint from $\mathcal{E}_{0}^{2}:=\left\{\eta^{2}\right.$ : $\left.\eta \in \mathcal{E}_{0}\right\}$. Then for $f \in L^{1} \cap L^{2}$

$$
\varphi(H) f(x)=\int_{-\infty}^{\infty} K(x, y) f(y) d y
$$

where $K=K_{a c}+K_{p}$,

$$
K_{a c}(x, y)=(2 \pi)^{-1} \int_{-\infty}^{\infty} \varphi\left(\xi^{2}\right) e(x, \xi) \overline{e(y, \xi)} d \xi
$$

and

$$
K_{p}(x, y)=\sum_{k} \varphi\left(\lambda_{k}\right) e_{k}(x) \bar{e}_{k}(y)
$$

Remark 1. If $|e(x, \xi)| \leq C$, a.e. $(x, \xi) \in \mathbb{R}^{2}$, then, under the same condition the integral expression (3) is valid for any $\varphi \in C(\mathbb{R})$ with compact support.

Remark 2. When $\varphi$ is smooth with rapid decay and $V$ is compactly supported in $\mathbb{R}^{3}$, a formula of this type appeared in $[9]$ by Tao.

\section{REFERENCES}

[1] P. Alsholm, G. Schmidt, Spectral and scattering theory for Schrödinger operators, Arch. Rational Mech. Anal. 40 (1971), 281-311.

[2] J. J. Benedetto, Harmonic Analysis and Applications, CRC Press, Inc., Boca Raton. FL, 1997.

[3] J. J. Benedetto and S. Zheng, Besov spaces for the Schrödinger operator with barrier potential, submitted.

[4] M. Christ and A. Kiselev, One-Dimensional Schrödinger operators with slowly decaying potentials: spectra and asymptotics, or, Baby Fourier Analysis Meets Toy Quantum Mechanics, Notes for IPAM tutorial, 2001 Workshop on Oscillatory Integrals and Dispersive Equations.

[5] C.-A. Guerin, M. Holschneider, Time-dependent scattering on fractal measures, J. Math. Physics 39(8), 1998.

[6] T. Ikebe, Eigenfunction expansions associated with the Schrödinger operators and their applications to scattering theory, Arch. Rational Mech. Anal. 5 (1960), 1-34. (Erratum, Remarks on the orthogonality of eigenfunctions for the Schrödinger operator on $\mathbb{R}^{n}$, J. Fac. Sci. Univ. Tokyo Sect.I 17, 1970)

[7] M. Reed and B. Simon, Methods of Modern Mathematical Physics III: Scattering Theory, Academic Press, New York, 1979.

[8] B. Simon, Quantum Mechanics for Hamiltonians Defined as Quadratic Forms, Princeton University Press, Princeton, New Jersey, 1971. 
[9] T. Tao, Scattering for the 3D Schrödinger equation with compactly supported potential, Preprint.

[10] D. Thoe, Eigenfunction expansions associated with Schrödinger operators in $\mathbb{R}^{n}, n \geq 4$, Arch. Rational Mech. Anal. 26 (1967), 335-356.

Department of Mathematics, Louisiana State University, Baton Rouge, LA 70803

E-mail address: szheng@math.1su.edu

$U R L$ : http://www.math. Isu.edu/ szheng 